\title{
Congenital Double-Orifice Mitral Valve with Mitral Regurgitation due to Flail Leaflet in an Elderly Patient
}

\author{
Shin-Jae Kim, M.D., Eun-Seok Shin, M.D. and Sang-Gon Lee, M.D. \\ Department of Internal Medicine, Ulsan University Hospital, \\ University of Ulsan College of Medicine, Ulsan, Korea
}

\begin{abstract}
We report here on a case of double-orifice mitral valve with mitral regurgitation in a 75-year-old female who had complaints of mild dyspnea. Transthoracic and transesophageal echocardiography showed two orifices that were supplied by their own chordae from a different papillary muscle. Color Doppler echocardiography revealed moderate to severe mitral regurgitation due to the flail posterior leaflet of the anterolateral orifice. Except for the persistent left superior vena cava, no other congenital anomaly was demonstrated. The patient became asymptomatic with the administration of angiotensin-converting enzyme inhibitor and diuretics, and she has been scheduled for long term follow-up.
\end{abstract}

Key Words : Double-orifice mitral valve; Mitral valve insufficiency; Echocardiography, Transesophageal

\section{INTRODUCTION}

A double-orifice mitral valve (DOMV) is a rare congenital malformation of the tensor apparatus such as the chordae tendineae and papillary muscles". Since this was first described by Greenfield in 1876, more than 200 cases of DOMV have been reported ${ }^{2}$. DOMV rarely occurs as an isolated anomaly: this disease is generally associated with a variety of other cardiac anomalies such as coarctation of the aorta, a bicuspid aortic valve, Ebstein's anomaly, and most commonly, atrioventricular septal defects, ${ }^{1,3}$. There are various hemodynamic effects for DOMV and the functioning of mitral leaflets is normal in most cases, but these leaflets can be regurgitant or stenotic ${ }^{4}$. We present here an unusual case of a 75-year-old woman who had congenital DOMV that was accompanied by mitral regurgitation due to the flail leaflet. To our knowledge, this is the first described case of DOMV with a flail leaflet in Korea.

\section{CASE REPORT}

A 75-year-old woman was referred to our hospital because of her mild exertional dyspnea and cardiac murmur. She had a long history of hypertension: 5 years earlier, she underwent a coronary angiographic study at our hospital because of her chest pain; no significant stenosis was found except for some minimal narrowing that was noted at the middle portion of the left anterior descending artery. During the same period, the precordial echocardiographic study revealed a slight left atrial enlargement and mild mitral regurgitation, but the quality of imaging resolution was poor and any further evaluation was not performed. The patient reported that she had been doing well until a few weeks earlier when she started suffering from a shortness of breath during mild exercise. Three days before her referral to the hospital, she had visited a general practitioner to get an evaluation of her symptoms. The doctor heard the murmur during the examination of the heart and he found blunting of the right costophrenic angle on the chest X-ray, so he referred her to us for further evaluation.

At the time of her second presentation, the patient had light dyspnea and a harsh pansystolic murmur (grade 3/6) was heard at the apex of the heart. The blood pressure was 140/80 $\mathrm{mmHg}$. The EKG showed a sinus rhythm with nonspecific ST-T

- Received : December 30, 2004

- Accepted : February 28, 2005

- Correspondence to : Sang-Gon Lee, M.D., Department of Internal Medicine, Ulsan University Hospital, 290-3 Cheonha-dong, Dong-gu, Ulsan, 682-714, Korea Tel : 82-52-250-7029, Fax : 82-52-251-8235, E-mail : sglee@uuh.ulsan.kr 

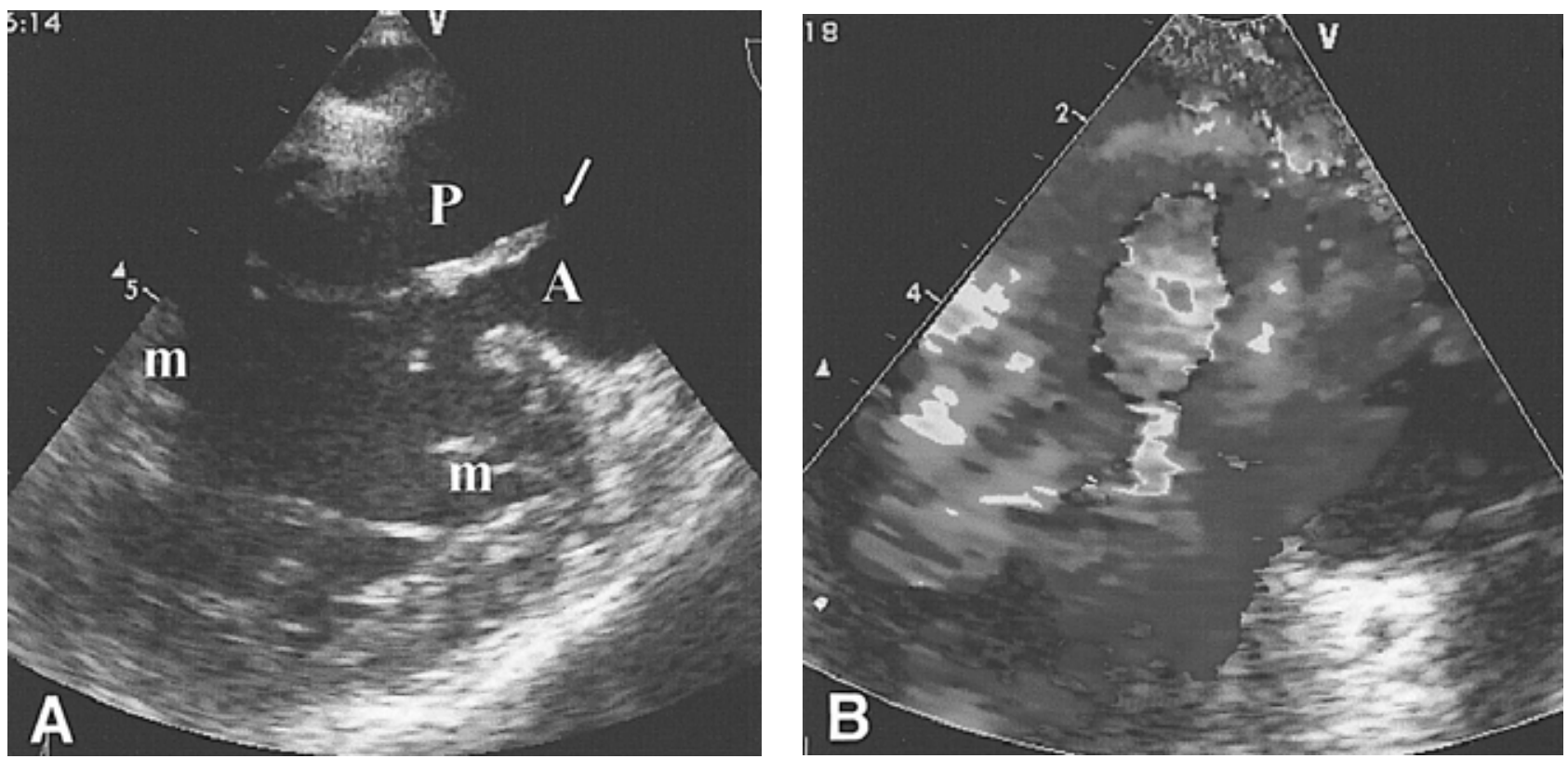

Figure 1. Transesophageal view of the two separated mitral orifices at $82^{\circ}$. (A) The fibrous bridge (arrow) divides the mitral orifice into the posteromedial $\mathrm{P}$; and the anterolateral $\mathrm{A}$; openings, and each orifice has its own chordae tendinae tethered to a different papillary muscle. (B) During diastole, the color flow image reveals a distinct mitral inflow jet flowing through each of the 2 orifices.

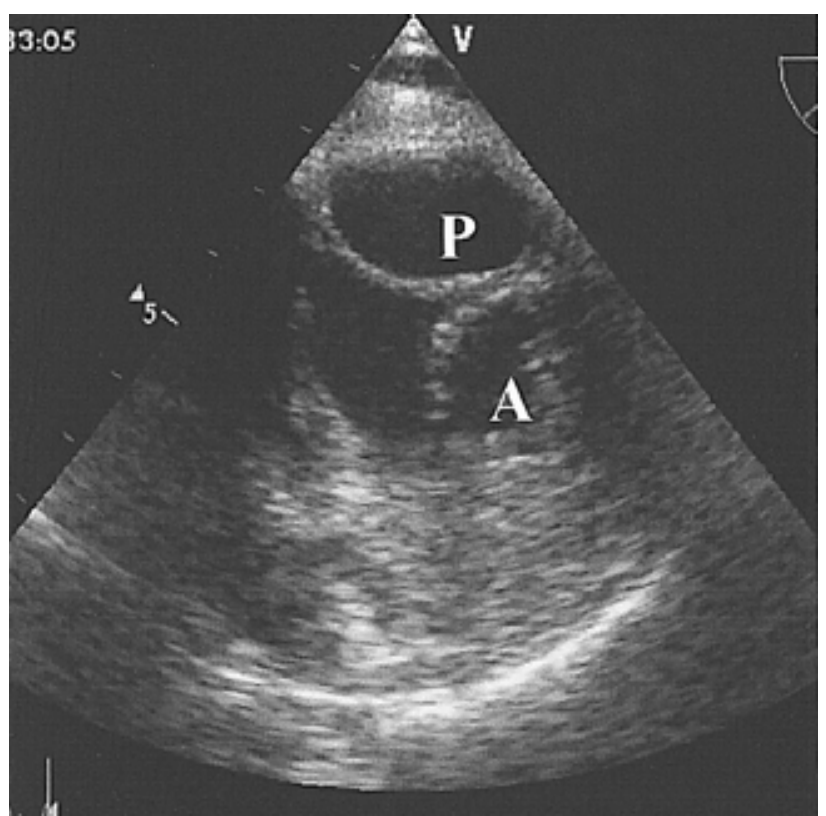

Figure 2. The transgastric view of the two mitral orifices at $37^{\circ}$. The larger posteromedial $P$; and smaller anterolateral $A$; orifices are clearly identified.

segment abnormalities. Echocardiography (Vivid 7, GE) revealed left ventricular hypertrophy and dilatation of the left atrium. The ventricle was not dilated and it had good contractility. There was also a very eccentric mitral regurgitation, but the morphology of the valve and the severity of the mitral regurgitation could not be precisely determined due to the poor image quality of the patient's test results.

Transesophageal echocardiography (TEE) clearly showed that the mitral valve was composed of two distinct orifices that were separated by a central fibrous bridge. It is interesting that each orifice had its own chordae tendineae tethered to a different papillary muscle (Figure 1A, 1B). The transgastric short axis view revealed a larger posteromedial orifice and a smaller anterolateral orifice (Figure 2). The posteromedial orifice was competent without any evidence of regurgitation or stenosis. At the level of anterolateral orifice, the color Doppler revealed moderate to severe eccentric regurgitation due to the flail posterior leaflet (Figure 3A, 3B). No other congenital cardiac anomalies were noted except for the persistent left superior vena cava that was demonstrated by injecting agitated saline at the antecubital vein of the left arm (Figure 4). The patient became asymptomatic with the administration of angiotensinconverting enzyme inhibitor and diuretics. She was scheduled for long term follow-up and prophylaxis was recommended for any potential infectious endocarditis.

\section{DISCUSSION}

DOMV consists of two anatomically distinct mitral orifices that are divided by an accessory bridge of fibrous connective tissue. The fibrous ridge is composed of the mitral leaflet tissue and chordae tendineae. The embryogenic origin of DOMV is 

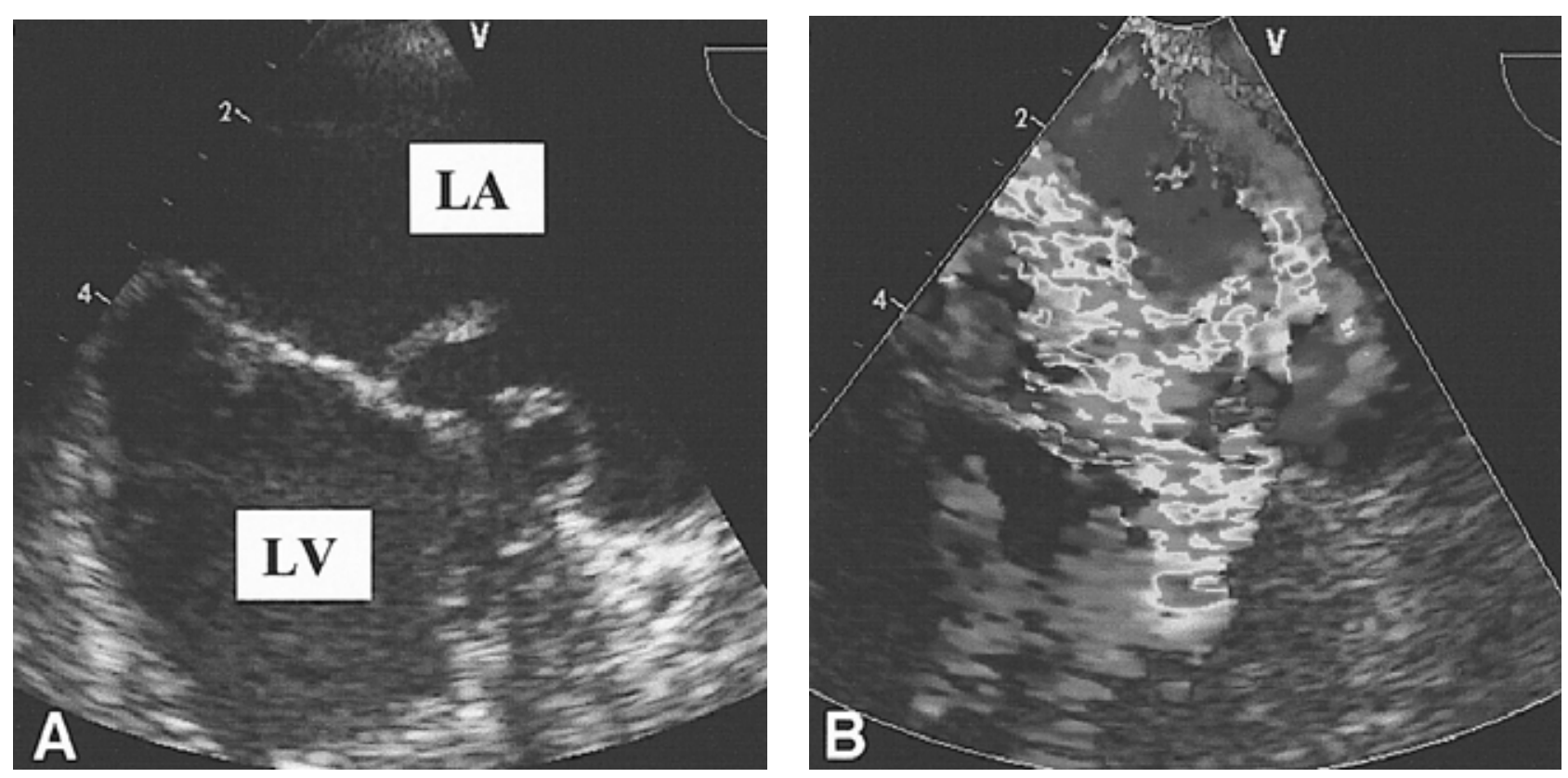

Figure 3. The transesophageal images taken from the long axis view of the left atrium and the left ventricle at $90^{\circ}$. (A) During systole, the flail posterior leaflet of the anterolateral orifice is demonstrated. (B) The color Doppler shows very eccentric mitral regurgitation at this site. LA; indicates left atrium; LV; indicates left ventricle

somewhat controversial. However, it is conceivable that the occurrence of abnormal differentiation of the mesenchymal endocardial cushion tissue into chordae instead of the leaflet tissue can result in such a defect".

The DOMV tensor apparatus is always abnormal and in the most common form, each orifice is separately attached to their individual, single papillary muscle, and this creates a kind of double parachute mitral valve, as was observed in our case. Trowitzsch et al. suggested it possible to distinguish three types of DOMV by sweeping the transducer in a cross-sectional view from the apex toward the base of the heart ${ }^{4}$. Our case could be classified as the complete bridge type in which two separate, complete orifices were seen at all levels of the mitral valve leaflets.

Although most of these cases are detected in childhood, DOMV has recently been reported on in adulthood or even in the elderly population, like our case ${ }^{5}$. In Korea, there are 3 case reports of DOMV, and two of these defects were found in children ${ }^{6,7)}$ and the other was found in a 42-year-old adult ${ }^{8)}$. Our current case is the first described case of DOMV in an elderly Korean patient, and this patient's heart defect was especially accompanied by the flail leaflet. To get a better picture of this defect, we used an appropriate precordial echocardiographic series of scan planes that were derived from various transducer positions; this series of scans can usually be readily obtained in pediatric patients and the high resolution of the images is generally observed. However, such good image resolution can rarely be obtained in adult patients. Thus, the

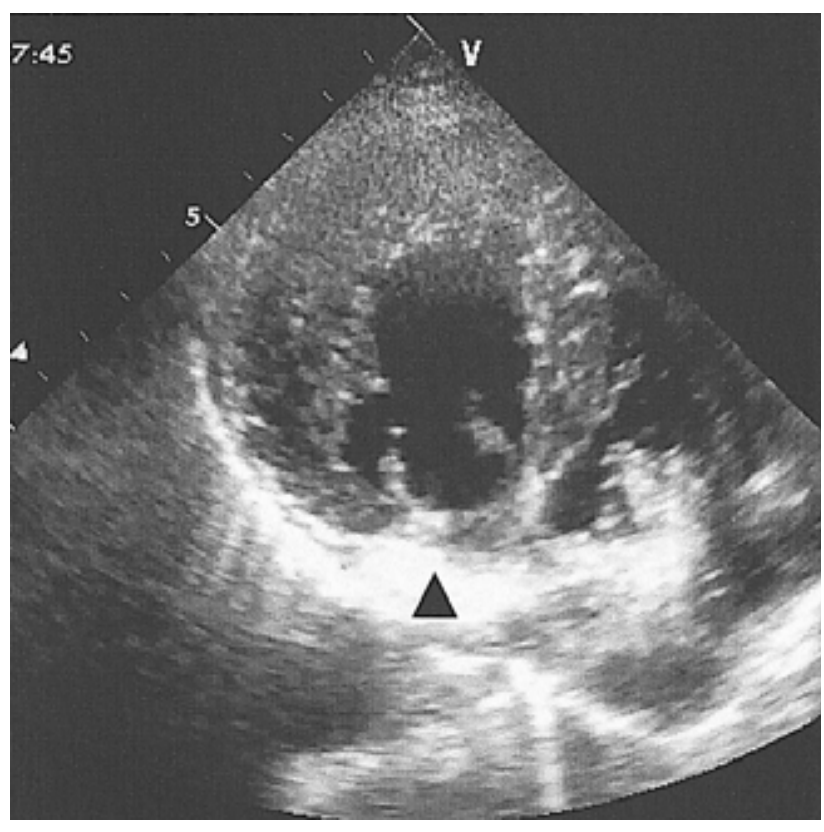

Figure 4. The transthoracic right ventricular inflow view. The air bubbles are visible in right atrium through the coronary sinus (A).

multiplane transesophageal approach with color flow mapping is superior to the precordial imaging for assessing the anatomical and functional status of this heart defect and for identifying any coexisting malformations, especially in adults or in the elderly patients $^{9-111}$. The usefulness of three-dimensional echocardiography for helping define the spatial location and the extent of 
the deformities in DOMV has been recently reported ${ }^{12)}$.

The clinical implications for this anomaly depend upon the severity of the regurgitation and also on the presence of any stenosis or associated malformations. The clinical features of this case suggest that this congenital DOMV had a relatively normal function for many years. Compared with the echocardiographic study that was conduced 5 years earlier, the mitral valve became flail later in life and it was recently aggravated to allow moderate to severe mitral regurgitation, and so the patient developed her symptoms. DOMV has an unusual importance to surgeons because it requires the physician to decide whether or not to replace or repair the valve, and DOMV is usually associated with other cardiac abnormalities. In spite of these difficulties, the mitral valve was successfully repaired in two similar reported cases of DOMV with flail leaflet ${ }^{13,14)}$. One case of DOMV with severe mitral regurgitation due to a partial flail leaflet was recently reported, and in this case the mitral valve was replaced with a mechanical prosthesis ${ }^{15}$. In our case, the patient had a history of hypertension and there was also some evidence of volume overload on the chest $X$-ray, and so we administered diuretics and angiotensin-converting enzyme inhibitor to lower the blood pressure and the filling pressures. The patient became asymptomatic and was scheduled for long-term follow-up.

This case demonstrates that careful examination is needed even in the elderly patients with mitral regurgitation, and if two separated orifices are present that are supplied by their own chordae from a different papillary muscle, then DOMV should be suspected and the patient should be assessed by using TEE. Further, this case remind us that DOMV is anatomically complex disease, it is commonly associated other cardiac abnormalities and a precise anatomical and functional assessment is required, especially in those cases in which surgical repair or valve replacement is needed.

\section{REFERENCES}

1) Bano-Rodrigo A, van Praagh $S$, Trowitzsch E, van Praagh $R$. Double-orifice mitral valve: a study of 27 postmortem cases with developmental, diagnostic and surgical considerations. Am J Cardiol
61:152-160, 1988

2) Greenfield WS. Double mitral valve. Trans Pathol Soc London 27:128-129, 1876

3) Sharma S, Loya YS, Daxini BV. Coarctation of aorta with unusual association of diverticulum of the left ventricle and double-orifice mitral valve. Int J Cardiol 30:113-115, 1991

4) Trowitzsch E, Bano-Rodrigo A, Burger BM, Colan SD, Sanders SP. Two-dimensional echocardiographic findings in double orifice mitral valve. J Am Coll Cardiol 6:383-387, 1985

5) Heyse AM, Vanhercke D, Nimmegeers J, Missault L, Claeys R, Hollanders G, Versee L. Mitral insufficiency with congenital double-orifice mitral valve in an elderly patient. Eur J Echocardiogr 4:334-335, 2003

6) Oh KC, Kim YW, Kim KB. Two cases of double-orifice mitral valve detected by echocardiography. J Korean Pediatr Soc 41:825-830, 1998

7) Park IS, Kim KS, Park YS, Hong CY, Yoo SJ, Song MG. Tricuspid atresia associated with double orifice mitral valve and coronary sinus septal defect. J Korean Pediatr Soc 34:1139-1145, 1991

8) Lee DI, Chung BY, Kim YW, Rim SJ, Ha JW, Chung NS. A case of isolated congenital double-orifice mitral valve. Korean Circ J 28:1007-1010, 1998

9) Hoffman P, Strumper O, Groundstroem K, Sutherland G. The transesophageal echocardiographic features of double-orifice left atrioventricular valve. J Am Soc Echocardiogr 6:94-100, 1993

10) Purnode $P$, Rombaut E, Gerard M, Alkhori M, Marchandise B. Double orifice mitral valve with flail leaflet: a transoesophageal echocardiographic examination. Eur J Echocardiogr 1:144-146, 2000

11) Mao JT, Tang J, Li Y, Sun P. Double-orifice mitral valve with multiple papillary muscles: a report of two patients. Angiology 50:771-775, 1999

12) Espinola-Zavaleta N, Vargas-Barron J, Keirns C, Rivera G, Romero-Crdenas A, Roldn J, Attie F. Three dimensional echocardiography in congenital malformations of the mitral valve. $J$ Am Soc Echocardiogr 15:468-472, 2002

13) Matsuaka R, Sakakibara T, Mitsuno M, Yagura A, Yoshikawa M, Ishikura F. Valve repair for mitral regurgitation associated with isolated double-orifice mitral valve. I Thorac Cardiovasc Surg 112:1666-1667, 1996

14) Tomita $Y$, Yasui $H$, Tominaga R. Mitral valve repair for isolated double-orifice mitral valve with torn chordae. Ann Thorac Surg 64:1831-1834, 1997

15) Shiran A, Lewis BS. Acquired partially flail leaflet causing severe mitral regurgitation in a congenital double-orifice mitral valve. J Am Soc Echocardiogr 17:478-479, 2004 